\title{
17 juin 1940, le plus grand désastre maritime Britannique?
}

\section{Éric Sinou-Bertault}

\begin{abstract}
2010 is the 70th birthday commemoration of a little known maritime catastrophe, which to this day is still mired in some secrecy. In June 1940, ahead of rapid German advances, allied troops in France organized a precipitous evacuation towards England. The troop transport Lancastria took on board thousands of soldiers and civilians. Attacked by the Luftwaffe, she sank in 24 minutes with high casualties. This event occurring on the day that France requested an armistice, Churchill, from now on alone vis-à-vis Hitler, blanketed this catastrophe in secrecy. Thanks to the testimony of many survivors and rescuers anxious to pay homage to the victims, we can reconstitute with precision this drama.
\end{abstract}

France 17 juin 1940, pour beaucoup ce jour ne signifie que la veille d'une date symbolique fondatrice de la Geste Gaullienne. Pour qui s'intéresse à l'histoire de la deuxième guerre mondiale, le 17 juin 1940, c'est le discours d'un vieux soldat qui, « le cœur serré, » annonce « qu'il faut cesser le combat. » ${ }^{1}$ Pour l'histoire maritime, cette date occultée recèle une des plus gigantesques tragédies navales: le naufrage du Lancastria. Ce drame méconnu d'une ampleur inégalée pour la marine Britannique est encore frappé du secret de la D-Notice ${ }^{2}$ jusqu'en 2040. Pourtant peu à peu et de plus en plus les rescapés, les témoins, ont voulu soulever the Iron Curtain ${ }^{3}$ que Sir Winston Churchill fit aussitôt descendre sur ce désastre anglais survenu à l'embouchure du plus français des fleuves.

Depuis trois jours déjà l'armée allemande est entrée dans Paris, la France est en débâcle, à Dunkerque l'essentiel du $\mathrm{BEF}^{4}$ en déroute vient de traverser la manche grâce au sacrifice de milliers de soldats français. Le reste des troupes Britanniques et Canadiennes cherche son salut en refluant vers les ports du Ponant. À l'estuaire de la

1 Allusion au discours radiophonique du maréchal Pétain nommé la veille chef du gouvernement et annonçant le 17 juin à $12 \mathrm{~h} 30$ qu'il faut cesser les combats.

2 Defence Advisory Notice déconseillant à la presse de diffuser des informations. Nicholas Wilkinson, Secrecy and the Media: The Official History of the D-Notice System (London: Routledge, 2009). Certains documents comme le rapport de mer du commandant du Lancastria sont toujours inaccessibles.

3 Allusion au discours de Churchill le 5 mai 1946 : « De Stettin sur la Baltique à Trieste sur l'Adriatique, un rideau de fer s'est abattu à travers le continent. »

4 British Expeditionary Force $=$ Corps expéditionnaire britannique en France.

The Northern Mariner/le marin du nord, XX No. 4, (October 2010), 407-418 
Loire que les forces allemandes sont sur le point d'atteindre, Saint Nazaire voit s'agglutiner une masse gigantesque de troupes Britanniques, Polonaises, Tchèques et Canadiennes grossie par la cohorte hagarde des réfugiés Belges et des marches de l'est. Harassés, accablés par la chaleur de ce si chaud mois de juin, harcelés par la Lutfwaffe, les civils, femmes, enfants se mêlent aux unités encore constituées qui se débarrassent de leurs équipements lourds, détruisant les munitions, le carburant, les véhicules et le matériel impossibles à ramener en Angleterre. Autour des routes, les fossés, les champs se remplissent de carcasses éparses, armée de fer fantomatique.

Depuis le début des opérations, la région Nantaise est la première base arrière britannique. L'essentiel de l'intendance s'y trouve à présent, les unités de soutien de la R.A.F. et depuis le début du mois, le nerf de la guerre, le commandement Pay Office $e^{5}$ est installé à Pornichet. La France terrassée est sur le point de capituler, il y a une semaine encore Churchill a voulu rassurer Paul Reynaud ${ }^{6}$ par un télégramme: "The maximum possible support is being given by British Forces in the great battle which the French Armies are now conducting with such undaunted courage. ${ }^{7}$ La veille à $10 \mathrm{~h} 15$, un premier War Cabinet présidé par Churchill évoque l'entrevue du secrétaire à la guerre Anthony Eden avec le général Charles de Gaulle et le projet d'un « Réduit Breton. » Une tête de pont serait ainsi maintenue dans l'ouest de la France avec un front tenu de Saint Malo à Saint Nazaire. ${ }^{8} \quad$ Pour les généraux français Maxime Weygand ${ }^{9}$ et Alphonse George $^{10}$ l'opération nécessitant au moins dix divisions et, ne couvrant pas Nantes, est irréaliste. $^{11}$ À $15 \mathrm{~h} 00$ un second War Cabinet propose un incroyable projet d'Union Franco-Anglaise, surréaliste pour qui connaît les particularismes des deux nations.

«France and Great Britain shall no longer be two nations but one. » Chaque citoyen français devient immédiatement citoyen britannique, chaque sujet britannique devient citoyen français, une seule monnaie commune, plus de frontière entre les deux pays, union des deux parlements, un seul commandement militaire. "And thus we shall conquer. ${ }^{12} \mathrm{C}^{\prime}$ est lyrique, beau comme l'antique mais si utopique!

À Saint Nazaire depuis le 12 juin la guerre est bien réelle. Un premier bombardement de nuit près de la centrale électrique déclenche d'énormes incendies, les dégâts sont lourds mais aucune victime encore. Puis l'aviation allemande se fait plus pressante, dans la nuit du 15 au 16 des mines magnétiques sont larguées dans l'estuaire. Les rares dragueurs de mines français sur zone s'activent pour enlever ces charges

5 Témoignage du Capt. C. Brooke, Pay Office.

6 Paul Reynaud (1878-1966), chef du gouvernement Français depuis le 18 mars 1940.

7 War Cabinet 170(40), 10 juin 1940, The National Archives, CAB/65/7/55.

8 Les anglais ont déjà dressé un tableau des besoins en charbon, laine, etc.. indispensable à une population estimée à 4 à 5 Millions. War Cabinet memorandum, 17 juin 1940, The National Archives, CAB/67/7/5.

9 Maxime Weygand (1867-1965), Généralissime depuis le 17 mai 1940.

10 Alphonse Georges (1875-1951), chef des opérations nord-est, l'essentiel de l'armée française.

11 War Cabinet 168(40), 16 juin 1940 - 10h15, The National Archives, CAB/65/7/63.

12 War Cabinet 170(40), 16 juin 1940 - 15h00, The National Archives, CAB/65/7/64. 
menaçantes, la Courbe saute sur l'une d'elles en emportant quatre marins, des dragueurs britanniques équipés du système $\mathrm{LL}^{13}$ viennent en renfort sans efficacité. Des dizaines de navires de transport et d'escorte se présentent pour sortir les troupes alliées de la nasse que les allemands sont en train de nouer. À l'aube du lundi 17, l'imposant $\mathrm{HMT}^{14}$ Lancastria long de 168 mètres et large de 21 arrive sur rade. Monsieur Guillemet, le pilote de Loire ${ }^{15}$ grimpe à la timonerie du paquebot et conseille au commandant Sharp de ne pas s'engager dans le chenal encore piégé. Vers $06 \mathrm{~h} 00$ l'ancre est jetée à la bouée de la Lambarde et ne sera jamais relevée, le navire vient d'ancrer son destin. Depuis son lancement vingt ans plus tôt en Écosse, ses turbines à vapeur de 13.500 chevaux-vapeur alimentées par des soutes de 1380 tonnes de mazout ont emmené jusqu'à 17 nœuds les 16.243 tonnes du paquebot dans bien des baies et des ports de l'atlantique, de méditerranée et de la mer du nord. Coiffé d'une unique et imposante cheminée c'est un navire élégant et confortable comme savaient l'être les palaces des années folles. La clientèle américaine peine à prononcer son nom originel Tyrrhenia, le 5 février 1924 il prend celui définitif

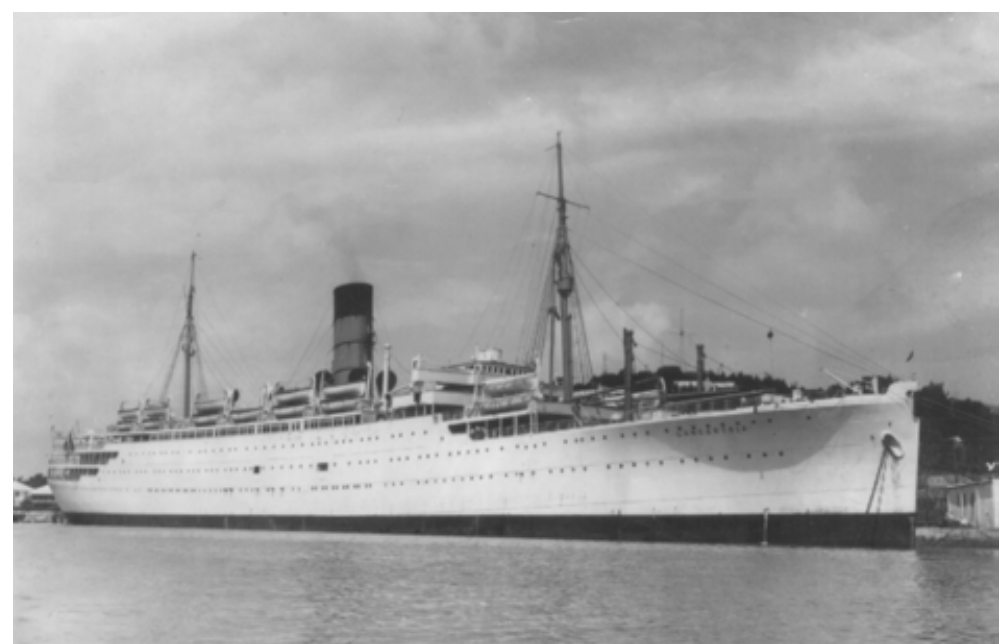

Illustration 1: Le Lancastria au temps de sa splendeur, environ 1928. Source: Lancastria Association, Scotland.

de RMS Lancastria. Pour un marin superstitieux changer de nom porte malheur, hélas le destin va alimenter la superstition. Entre 1924 et 1930, entre l'Angleterre, la France, l'Allemagne et l'Amérique il traversera 55 fois l'Atlantique; avant d'emmener dans ses sept ponts les passagers privilégiés des 580 cabin-class et les mille de troisième classe, croiser en méditerranée ou aux Bahamas. Aujourd'hui sa mine est plus terne. À la déclaration de guerre il relie pour sa dernière croisière Liverpool à New-York. Dans le port de l'Hudson il se transforme pour la guerre, sa coque est repeinte en gris, ses hublots occultés, son mobilier, sa décoration enlevés. Le 13 avril 1940, le HMT Lancastria est mis à la disposition de la Royal Navy. L'équipage a reçu une formation très sommaire de navigation en convoi et d'exercice d'abandon; et à la poupe et à la proue le pont est garni d'un canon et de mitrailleuses.

Les alliés ayant décidé de contrer Adolf Hitler en Norvège, le Lancastria quitte

13 LL, ou Double Longitudinal sweep (drague longitudinale double) devenue opérationelle en mars 1940. Eric Grove, Defeat of the Enemy Attack on Shipping (London, 1957), p. 192.

14 His Majesty's Troopship.

15 Les pilotes de Loire sont chargés de guider les navires dans l'estuaire de la Loire. 
Glasgow le 14 mai rempli de troupes pour Narwick. L'opération stratégique tourne court pour les alliés et le 28 mai ce sont 2583 britanniques, français, canadiens et polonais que le navire ramène aux îles d'Orcades, ${ }^{16}$ un avion allemand lui décoche une salve de bombes mais le manque, il vient d'essuyer sa première attaque. Dirigé sur Liverpool pour un carénage, il appareille d'urgence pour la France quittant pour toujours son port d'attache. À Plymouth, rejoint par les paquebots Franconia $^{17}$ et Oronsay, ${ }^{18}$ il fait route vers Brest. Du large, la vision du port breton est apocalyptique, ses réservoirs d'essence sont en feu. Le convoi, guidé par un remorqueur, descend vers les côtes du Morbihan. Un avion sort de la brume, le Franconia est sa cible; coup au but, le pont du navire est à fleur d'eau, pour lui la mission s'achève et il entame un périlleux retour à Liverpool. ${ }^{19}$

À 03 h50 le 17 juin, l'ambassadeur Britannique à Bordeaux transmet à Londres la composition du nouveau gouvernement français présidé par le maréchal Philippe Pétain. Le War Cabinet $^{20}$ réuni en fin de matinée comprend que, inévitablement, la France va jeter le gant et s'inquiète du sort de l'encore puissante marine française. La nomination au ministère de la Marine de l'amiral François Darlan qui a toujours déclaré qu'il ne livrerait pas la flotte aux allemands rassure un peu. ${ }^{21}$. La veille, le télégramme No. 368 DIPP adressé à Paul Reynaud a conditionné l'acceptation anglaise d'un armistice français au ralliement de la flotte française dans les ports britanniques. Reynaud évincé du gouvernement a-t-il transmis ce message? Sir Ronald Campbell, ambassadeur en France est chargé de le communiquer personnellement à Pétain. Contre l'avis du général John Dill, chef d'état-major Impérial, il est décidé que le francophile général Alan Brooke qui a remplacé John Gort à la tête du $\mathrm{BEF}^{22}$ reste en France pour procéder à l'évacuation des 50.000 hommes encore présents sur le continent. À 12h40, en plein conseil, la nouvelle tombe: le maréchal Pétain vient de faire son discours sur les ondes annonçant aux français qu'il faut cesser le combat.

Mouillé par vingt mètres de fond à quatre miles du rivage le Lancastria commence l'embarquement des passagers de sa funeste croisière immobile. Alors que le dragage des mines se poursuit, trois contre-torpilleurs, trois gros remorqueurs et une noria de petites unités déversent leurs flots d'évacués. Les premiers accueillis avec méthode reçoivent un ticket d'embarquement, un gilet de sauvetage et se dirigent en bon ordre vers les zones allouées. Les officiers et gradés disposent des cabines et sont traités par les stewards comme la Cunard traite ses passagers habituels. Un breakfast très anglais leur est offert. Les passagers d'infortune qui marchent et dorment sur les routes depuis des jours semblent déjà arrivés en Angleterre. Le salon de coiffure est un des endroits les

16 Orkney Islands, nord de l'écosse.

17 Franconia, $190 \mathrm{~m}$ de long, $20341 \mathrm{t}$.

18 Oronsay, 210m de long, sera torpillé le 09 septembre 1942 au large du Liberia.

19 Il y parviendra, continuera son service et servira de Q.G. lors du sommet de Yalta.

20 War Cabinet 170(40), 10 juin 1940, The National Archives, CAB/65/7/55.

21 Télégramme de Lord Campbell reportant son entrevue avec l'amiral Darlan (No. 435 DIPP).

22 Brooke se trouve alors à Redon à $60 \mathrm{~km}$ au nord de St Nazaire. Alan Francis Brooke (18831963) né en France à Bagnères-de-Bigorre sera promu maréchal en 1944 : Pierre Montagnon, Dictionnaire de la seconde guerre mondiale (Paris: Pygmalion, 2008). 
plus prisés, les hommes qui pour beaucoup n'ont pas eu de permission depuis 1939 en profitent pour rectifier leur mine avant de rentrer au pays. L'on soigne ses ampoules, cherche le repos, c'est le calme avant la tempête de feu qui va venir du ciel. Harry Grattidge, le commandant en second supervise l'embarquement « Toute la matinée le ciel vibra du bruit des avions, voix froide et mécanique de la colère du vingtième siècle. ${ }^{23}$ À présent, la salle à manger et son personnel stylé propose un déjeuner gastronomique inespéré à tous ces civils et militaires affamés qui se contentent de corned-beef ou de biscuits depuis des jours «Un délicieux repas nous fut servi très élégamment par les serveurs, $\gg^{24}$ la carte bien différente d'une cantine militaire.

Hors de cet intermède pantagruélique, le flux des embarqués grossit furieusement. Chaque contre-torpilleur apporte un millier de passagers d'un coup, les remorqueurs prés de quatre cents. Un premier pointage dépasse déjà les 5000 passagers alors que le navire est prévu pour 3000, le Second s'en inquiète, il ferme le sabord à l'arrivée d'un nouveau contre-torpilleur qui lui tire une bordée d'insultes avant de se diriger dépité vers l'Oronsay.

Le Lancastria déborde d'hommes transpirants confinés dans leurs tenues kakis, serrés, entassés, se coudoyant; les ponts, les coursives s'engorgent, les officiers se retrouvent à plusieurs par cabine. Depuis longtemps il n'y a plus de gilet à distribuer. « Aux alentours de $15 \mathrm{~h} 00$, j'étais de quart dans l'équipe de sécurité qui se trouvait sur le pont, la capitaine Sharp me demandait d'aller avec mon compagnon vérifier le nombre de personnes embarquées, l'évaluation était de plus de 7000 ne comprenant pas l'équipage qui totalisait à lui seul 450 personnes. ${ }^{25}$ Arrivé le matin et enrôlé comme adjudantmajor chargé de recenser les hommes à bord le lieutenant-colonel F.E. Griggs en compte d'avantage encore « suivant ma propre estimation, nous n'étions pas loin de 9000 soldats à bord? ${ }^{26}$

Né en 1880 à Liverpool dans une de ces familles de tradition maritime liées à la Cunard, ${ }^{27}$ le commandant Rudolph Sharp est de plus en plus anxieux: " Homme corpulent et solennel qui avait horreur de la guerre. Ses nerfs étaient plus tendus qu'il n'aurait fallu à un bon et consciencieux marin qui n'a jamais songé à la mort. » ${ }^{28}$ Il sait qu'il offre un cible immanquable à l'aviation ennemie qui rode et au large les U-Boot guettent. Il veut partir en convoi avec l'Oronsay, demande par signaux à un contretorpilleur sa protection, la réponse ne viendra jamais.

Vers 14 h00, attiré sans doute par ses doubles cheminées un bombardier allemand lâche quatre bombes sur l'Oronsay. Sa timonerie est entièrement détruite, l'explosion courant les coursives fauche beaucoup de monde, arrache un canot de sauvetage mais

\footnotetext{
23 Commodore Grattidge.

24 Soldat P.H. Fairfax.

25 Michael Sheelan, membre Canadien de l'équipage.

26 Lt.- Col. F.E. Griggs.

27 La plus importante compagnie maritime britannique qui arma aussi le Titanic, le Lusitania, et dernièrement le Queen Mary II.

28 Commodore Grattidge.
} 
aucune partie vitale n'est touchée. Très britanniques, au restaurant des officiers attendent la fin de l'alerte pour que le steward leur apporte leur deuxième plat, sur le pont le Captain H. Fuller prend le thé, pour un anglais c'est l'heure! ${ }^{29}$

15h45, nouvelle alerte, à basse altitude plusieurs bombardiers attaquent le Lancastria. $^{30}$

15h48, une première bombe explose dans la cale No.1 où sont entassés depuis le matin plus de 800 hommes de la RAF qui n'ont pas une chance. Sur le pont les mitrailleuses antiaériennes tentent d'atteindre les porteurs de mort qui prudents arrivent dos au soleil, ce si chaud soleil de juin 40. Une deuxième bombe frôle la cheminée et tombe dans la salle des machines. À la passerelle, le commandant appelle les mécaniciens, le transmetteur d'ordre ne répond pas, le Lancastria est irrémédiablement immobilisé. Un troisième projectile au ras de l'eau longe la coque et l'éventre, 1400 tonnes de mazout vont vomir de ce trou béant. Compressé dans les coursives on cherche à sortir du piège d'acier, des soldats s'escamotent pour laisser passer les nombreuses femmes et enfants. Des officiers au réflexe militaire cherchent à maintenir l'ordre : « Il y avait un officier avec un revolver obligeant les hommes à descendre ne réalisant pas que nous étions en train de sombrer. " ${ }^{31}$ Touché à mort, ébranlé par les explosions, croulant sous sa cargaison humaine, le Lancastria commence lentement à pencher sur tribord. Le Commandant en second hurle: « Tout le monde à bâbord! Dégagez les canots à bâbord » le gîte change de côté et pèse à présent aussi dangereusement sur bâbord. Un nouvel ordre fuse "Otez tous vos chaussures! " l'on obéit, beaucoup commencent à se déshabiller conscients qu'il va falloir affronter l'océan. Hormis l'équipage peu de marins sont à bord. Civils, infirmières, religieuses, armée du salut, aviateurs, sapeurs, artilleurs, fourriers...combien connaissent les procédures d'abandon d'un navire? combien savent nager? «Je vis un groupe de prêtres aumôniers de l'armée et du YMCA prier à genoux. ${ }^{32}$ Les canots sont amenés mais les manœuvres sont précipitées, plusieurs pleins se renversent ou largués trop haut éclatent en touchant la mer. ${ }^{33}$ Le navire donne de la bande rapidement, déjà des gens plongent, un des stewards dit à un jeune soldat.

Les gilets de sauvetage mal maintenus remontent brusquement en touchant la surface de l'eau et brisent les vertèbres. Pour beaucoup les « life-jackets » seront des instruments de mort, des centaines de ces victimes flotteront inertes parmi les survivants médusés. ${ }^{34}$ Les avions reviennent et déchargent leurs mitrailleuses, la D.C.A. réplique.

29 Capt. H. Fuller de l'ordre de l'empire britannique.

30 Les données concernant les assaillants sont confuses. Il pourrait s'agir de Stukas JU $87 \mathrm{du}$ VIII Flieger Korps et de Junker 88 de l'escadrille II/KG30 basés en Belgique. Un témoin affirmera même avoir vu un avion italien. Le rapport de guerre du 18 juin 1940 de la Kriegsmarine note: « situation aérienne: d'après message nocturne gros succès de l'assaut du 17 juin contre réunion de bateaux à l'embouchure de la Loire. Plusieurs gros transports, dont 1 de 30000 t coulé, autres dont 2 de 10000 t et 1 de 25000 t endommagés sérieusement ».

31 Major P.H. Fairfax.

32 Sergent Harry Pettit.

33 Sur 28 canots, un sur quatre sera amené et seulement quatre flotteront.

34 Les modèles de gilets distribués ne possédait pas de sous-cutale (sangle passant entre les 
«Sur le boulevard des engins militaires armés de canons antiaériens tiraient sur les avions dans un grand bruit de sirènes. ${ }^{35}$ Comme un gigantesque « culbuto, » le grand bateau se couche sur le flanc laissant impudiquement apparaître une hélice. Beaucoup de naufragés s'accrochent encore à la coque renversée. Par peur, par courage, par défi, par désespoir, des chants s'élèvent "Roll out the Barrel, » chanson de taverne, hymne à la patrie "There'll always be an England » ou cantique. Des centaines de poitrines crient qu'elles vivent encore.

16h02, 14 minutes après le premier impact l'immense cheminée du Lancastria s'enfonce et disparaît dans les eaux. Dix minutes plus tard, il ne reste plus rien du transatlantique gobé par l'océan. Vingt-quatre minutes auront suffit pour engloutir les 16000 tonnes emportant des milliers de corps, prisonniers de la coque ou aspirés dans ce maelström. Une couche de $20 \mathrm{~cm}$ de mazout épais surnage. Des milliers d'hommes, de femmes, d'enfants, luttent pour la survie, avalant le liquide noirâtre qui les étouffe, s'agrippant au moindre objet flottant. Les avions fignolent leur besogne, mitraillant les naufragés, tirant des balles incendiaires pour tenter d'enflammer le mazout. Ça et là, des flammes courent sur la mer. À chaque instant des têtes s'enfoncent et disparaissent dans les flots. " $\grave{A}$ un moment, je fus prés d'un groupe incluant deux femmes, l'une d'elles tenant un bébé, tous bientôt balayés. » ${ }^{36}$

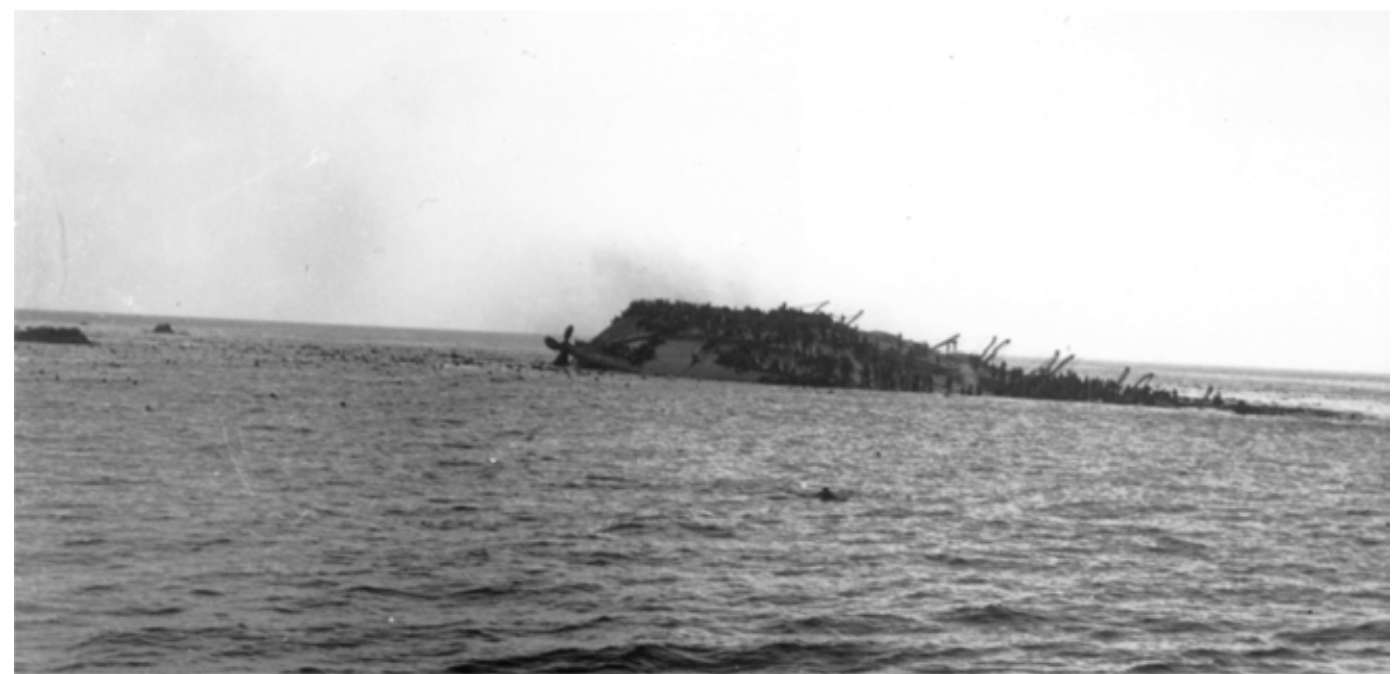

Illustration 2: Le naufrage vu de l'Oronsay; noter les naufragés entre l'arrière du Lancastria et les rochers à gauche. Source: Lancastria Association, Scotland.

Surnageant, survivant, cramponné, arc bouté, agriffé à un havresac, un aviron, une planche pour ne pas être emporté, un flot humain s'accroche à la vie. Certains renoncent, se laissent partir, ne peuvent résister à une crampe soudaine, au poison du mazout ingéré, à l'épuisement, au découragement. Les secours convergent, de dizaines

jambes).

35 Henri Frérel, 15 ans à l'époque (témoignage du 17 août 1993).

36 Sergent R. Wordsworth, Royal Army Ordnance Corps. 
d'embarcations des bras vigoureux hâlent les naufragés. Marins anglais, hommes de la Société Nationale de Sauvetage en Mer, du bac de Mindin, ${ }^{37}$ équipages des chalutiers, des remorqueurs, du bateau-feu, des pilotes de Loire, arrachent à la mer, à la mort, des corps englués et moribonds. Les rescapés en état de choc s'entassent dans les bateaux salutaires trop vite remplis. Il faut faire des tris inhumains, sauver en priorité ceux qui semblent les plus aptes à survivre, puis rejoindre la côte toute proche pour débarquer cette cargaison d'outre-tombe, puis revenir en sauver encore et encore, il y en a tant. Les survivants loueront ces gens de mer. À terre, consternés, débordés par l'ampleur du désastre les

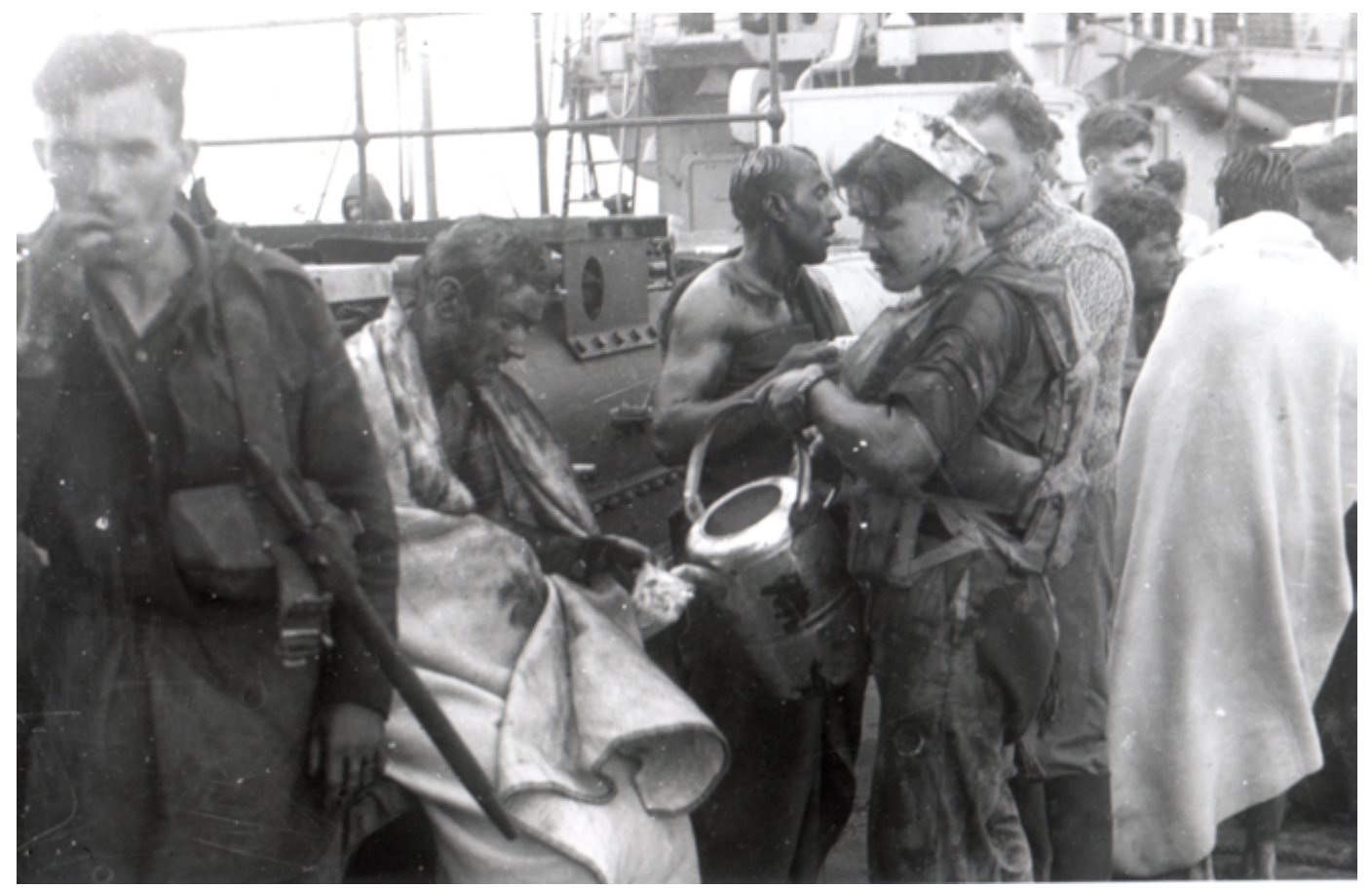

Illustration 3: Les rescapés. Source: Lancastria Association, Scotland.

secours se dévouent pour maintenir en vie les victimes grelottantes, intoxiquées par le pétrole, blessées, brûlées, poisseuses mais chanceuses malgré tout. À la toute fin du millénaire sur cette même côte, l'opinion à juste titre, sera choquée par une catastrophique marée noire ${ }^{38}$ mazoutant les oiseaux de mer. Qui se souviendra alors que soixante ans plus tôt des milliers d'hommes, de femmes, d'enfants avaient connu le même supplice? Les hôpitaux, infirmeries, écoles de Saint Nazaire sont bourrés de rescapés qu'il faut soigner, réconforter, réchauffer, nettoyer à l'essence ou enduire de beurre pour décoller ce maudit mazout. Et sur terre la guerre ne s'est pas arrêtée, les allemands se rapprochent, les rescapés doivent rejoindre l'Angleterre, trouver le courage d'embarquer encore sur une de ces cibles flottantes pour ne pas finir dans un stalag. l'Oronsay, rempli jusqu'à la

37 Le bac qui assure la navette entre les deux rives de l'embouchure de la Loire.

38 Le 12 décembre 1999, le pétrolier Erika sombre en rejetant sur les côtes bretonnes ses 37000 $\mathrm{t}$ de pétrole lourd. 
gueule meurtri mais naviguant, appareille; il n'a plus de timonerie, de salle de cartes, d'instruments. Son remarquable commandant naviguera avec une boussole de poche et ramènera à Plymouth 2500 évacués, parmi eux beaucoup de rescapés du Lancastria; la plus jeune, fille d'un couple de réfugiés Belges, a 30 mois.

L'estuaire se vide de dizaines de navires faisant route vers l'Angleterre. À minuit, le Gynafic et le Royal Ulsterman emportant 3500 hommes seront les derniers à quitter la zone. Deux jours plus tard alors que la Wehrmacht parade à Nantes, ${ }^{39}$ s'extirpant du chantier naval de Saint Nazaire, le cuirassé Jean Bart ${ }^{40}$ dont la construction n'est achevée qu'aux trois-quarts, parviendra par un sensationnel exploit maritime, à s'arracher des serres de l'aigle allemand. Le vendredi 21 , les premières bottes de la $11^{\circ}$ Schützen Brigade foulent le sol d'une ville qui durant cinq ans va devenir une des plus importantes bases de U-Boot. Les alliés devront attendre le 11 mai 1945 avant de libérer Festung Saint Nazaire ${ }^{41}$ dernière place forte d'Europe.

Dans la semaine qui suit le naufrage le commandant Sharp et son second rescapés se retrouvent à Liverpool: « ce fut dans un restaurant, avec comme musique de fond les rires venant du bar, que nous préparâmes le rapport officiel. $»^{42}$ Après un nouveau commandement sur l'Antonia, le commandant Sharp prend la barre du Laconia. Le 12 septembre 1942 il est torpillé par le U-156 au large de Dakar, pour lui un deuxième naufrage est insupportable; il veille à l'embarquement dans les canots puis s'enferme dans sa cabine et disparaît à jamais.

Longtemps le Lancastria va hanter le littoral, les jours suivants, la mer trop repue recrache ses morts. De la baie de La Baule à l'ile d'Yeu, ${ }^{43}$ des semaines durant, chaque marée laisse d'innombrables cadavres gonflés et informes sur les plages. Partout des cimetières s'improvisent. Le dernier cadavre surgira six mois plus tard. ${ }^{44}$ Les gens de la côte, connaissent trop bien la frustration des deuils impossibles des disparus en mer. Ils recueilleront pieusement sur les corps les objets, papiers détrempés, plaques à remettre aux familles après la guerre. Des civils bravant l'occupant fleuriront les tombes anglaises.

D'où venaient-ils? Anglais, Écossais, Canadiens, Néo-zélandais, Indiens, Sud Africains, Belges, Hollandais, Polonais...Combien de civils? D'enfants?

Combien de morts? Le nombre reste inconnu, 4000 au moins, 5000 probablement, 6000 peut-être, d'avantage? Calcul sordide mais dérisoire quand les cinq années suivantes vont compter les morts par dizaines de millions. Dans la fureur de la guerre un silence de plomb s'est abattu sur ce drame, il perdure. Le War Cabinet du

39 Nantes déclarée ville ouverte comme toutes les villes de plus de $20,000 \mathrm{~h}$ se trouve à $65 \mathrm{~km}$ de Saint Nazaire.

40 Cuirassé de $47500 \mathrm{t}$ long de $248 \mathrm{~m}$, fleuron de la flotte ralliera Casablanca.

41 Forteresse St Nazaire. Les bombardements alliés rasent la ville mais laisse la base sousmarine intacte.

$42 \quad$ Il est encore secret en 2010.

43 Par un curieux raccourci de l'histoire des victimes reposent au cimetière de l'île d'Yeu à quelques mètres d'une tombe portant juste: Philippe Pétain, Maréchal de France.

44 Repêché le 17/ décembre 1940 sur la plage du Verdon à l'embouchure de la Gironde. 
lendemain, en l'absence de Churchill, mentionne brièvement le naufrage du Lancastria avec 3000 hommes à bord et un nombre de victime inconnu. Mais le 18 juin à Londres l'obsession est le sort de la flotte de Darlan qui est sûrement l'homme du jour ${ }^{45}$ devant De Gaulle que l'on autorise néanmoins à lancer un appel à la BBC.

Churchill informé du sort du Lancastria impose un black-out total à la presse «The newspapers have got quite enough disaster for today, at least. » Sa démarche semble légitime à l'heure où l'Angleterre se retrouve seule au combat face à une Allemagne triomphante partout; il doit maintenir le moral de son peuple. Les pertes du $\mathrm{BEF}^{46}$ sont énormes, politiquement Churchill est encore fragile et peut-être sent-il planer l'ombre du désastre naval des Dardanelles. ${ }^{47}$ Dans ses mémoires évoquant le naufrage du navire transportant 5000 hommes dont 3000 périrent il explique qu'il voulait garder l'information secrète quelques jours puis " forgot » de lever l'interdiction. "I had intended to release the news a few days later, but events crowded upon us so black and so quickly that I forgot to lift the ban, and it was some years before the knowledge of this horror became public. $\gg^{48}$

Ce n'est qu'à la fin 1941 que les familles recevront les télégrammes fatidiques annonçant les décès, beaucoup n'auront jamais de nouvelles de leurs disparus. Le 26 juillet 1940, la presse anglaise tente de lever le voile, le Daily Mirror titre: « Liner Sunk after Nazi Pledge » et annonce 2843 disparus. La semaine suivante c'est au tour du New York Sun de faire timidement état de 600 victimes sur les 6000 passagers. Le 20 août entre une publicité pour Colgate et Coca-Cola, le magazine américain Life publie comme «picture of the week» deux photos du naufrage en estimant les victimes à $2800 .{ }^{49} \mathrm{Et}$ puis c'est le silence pour longtemps. "On nous a interdit sous les Kings Regulations de mentionner quoique ce soit sur le Lancastria. » Dans les musées et la colossale littérature consacrés à cette guerre même les historiens les plus reconnus n'évoquent jamais cette affaire, les exceptions sont rarissimes. Manifestement le fantôme du Lancastria dérange encore. Pourquoi?

Le navire n'a pas bougé. « La partie avant tournée vers St Nazaire semble intacte, l'épave est très dangereuse, tout le côté tribord muni d'une rangée de hublots est encore debout... une cassure au milieu du navire. ${ }^{50}$ Longtemps des plongeurs raconteront la vision d'épouvante de ces milliers d'ossements encagés. Une balise rouge signale l'épave, peint au pochoir un nom s'y détache: Lancastria. En 2006 le site devenu sanctuaire, la

45 Il est demandé au Premier Lord de l'amirauté de rester à Bordeaux auprès de Darlan. War Cabinet 171(40), 18 juin 1940, The National Archives, CAB/65/7/66.

46 Rapport War Office: pertes totales 66008 hommes et 2972 officiers, The National Archives, WO222/1532.

47 Premier Lord de l'Amirauté durant la première guerre mondiale, il lui fut durement reproché les revers des Balkans.

48 Winston Churchill, The Second World War, Vol. II : Their Finest Hour (London : Cassell, 1949), 172.

49 Life, 9, no. 8 (19 août 1940).

50 Témoignage des plongeurs Foulloneau et Meigen. 
plongée sous-marine est interdite sous un cercle d'un rayon de 200 mètres. ${ }^{51}$

Parvenus à l'age mûr des survivants éprouvent le besoin de briser le silence et de parler. Le Major Harry Pettit retrouve des rescapés et collecte les témoignages pour la rédaction d'un livre. Avant d'accomplir son devoir de mémoire il décède en 1971 et son travail est repris par John L. West. ${ }^{52}$ Une association anglaise est créée et les premiers pèlerinages de rescapés et de familles de disparus se rendent à Saint Nazaire tous les 17 juin. Un sobre cénotaphe de granit est érigé sur le front de mer face au lieu du drame.

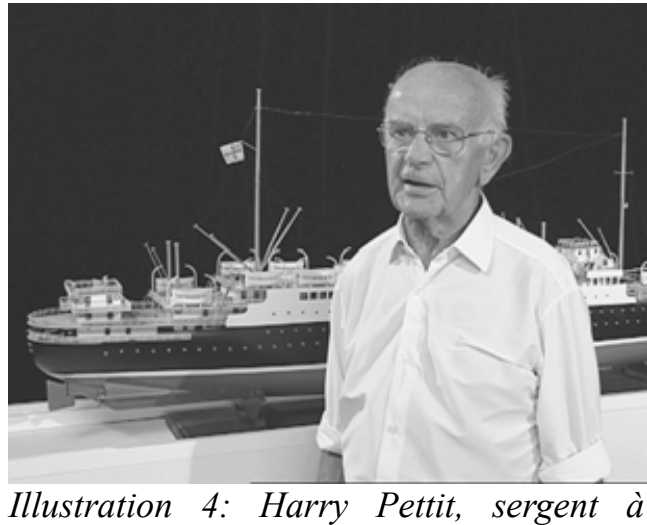

l'époque du naufrage, devant la maquette $d u$ Lancastria, 2005. Source: Lancastria Association, Scotland.

Petit-fils d'un rescapé, Mark Hirst a créé la Lancastria Association of Scotland et se démène pour faire connaître et reconnaître la tragédie. Il alimente un site internet et œuvre auprès de son gouvernement. En France, son ami Yves Beaujuge, capitaine du bateau pilote de Loire La Couronnée II se dévoue depuis dix ans pour honorer la mémoire des victimes, renseigner les familles et recenser avec une patience monacale les tombes, les témoignages locaux et étrangers, les documents. N'attendant pas que James

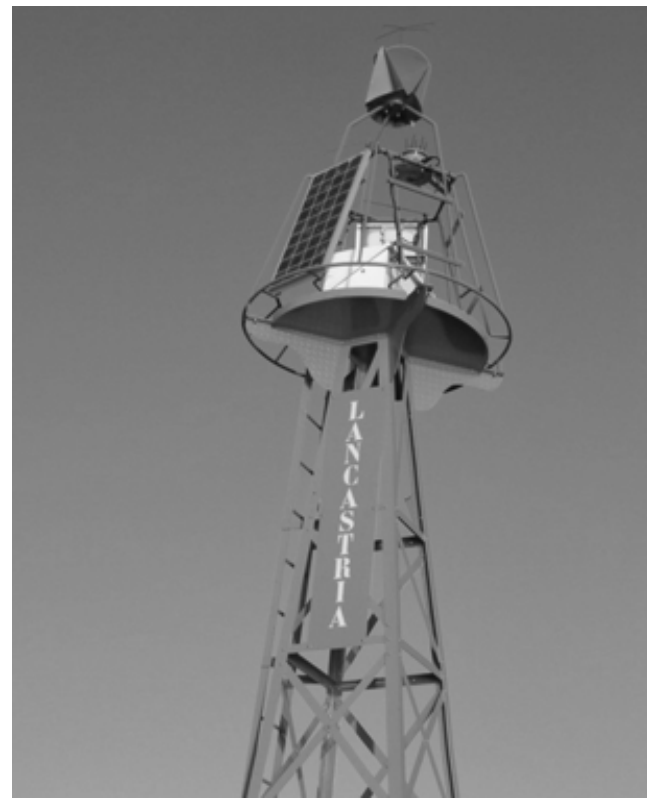

Illustration 5: La bouée signalant l'épave du Lancastria. Photo Yves Beaujuge.
Cameron s'intéresse à cet Avatar maritime, il participe en 2008 à la création d'un excellent DVD de 52 minutes réalisé par Christophe François pour France 3 Ouest. Il donne des conférences et vient d'écrire le premier livre français consacré à cette histoire. Yves Beaujuge n'est pas parent de victime, ni historien, ni écrivain, ni anglais, c'est juste un homme de mer touché par un drame de mer.

Décédé au début de 2010 Joseph Steenway, canadien réchappé, ne savait pas nager, il dû son salut à une planche qui surnageait. Pour le 70ième anniversaire, les survivants clairsemés entourant sa fille accompagneront en mer prés de la sépulcrale bouée rouge la dispersion de ses cendres, la mer toujours reprend ses droits..

$* * *$

Bibliographie: voir page suivante.

51 Arrêté no. 2006/14 du 03 mai 2006, signé par le V.A.E. Merrer.

52 L'essentiel des témoignages cités provient de ces sources. 


\section{Bibliographie:}

- Beaujuge, Yves. La tragédie du Lancastria 17 juin 1940. St Nazaire: 2010.

- Bertin, François. Saint Nazaire sous l'occupation, Rennes: Éditions Ouest France, 1989.

- Brauer, Luc. Saint Nazaire 1939-1940, Luc Braeuer éditeur : Batz sur mer, 2004.

- Boutin, Émile, Les grands naufrages de l'estuaire de la Loire, Siloé: Rennes, 1992.

- Mémoire et Savoir Nazairiens. 17 juin 1940/ 19 juin 1940 deux dates mémorables. St Nazaire, 1996.

- $\quad$ Petit, Denise, La vie quotidienne d'une Nazairienne de 1939 à 1944. St Nazaire, 2001.

- West, John L. traduit par Madeleine Jegu, Le destin tragique $d u$ paquebot Lancastria, témoignages. Madeleine Jegu, traducteur. St Nazaire : Mémoire et Savoir Nazairiens, 1996.

- DVD réalisé par Christophe François, Lancastria histoire d'un naufrage confidentiel, France 3 Ouest - Atlantic Télévision, 2008 - 52 minutes + bonus version Française et Anglaise.

- http://www.lelancastria.com (site Français)

- http://www.lancastria.org.uk (site Britannique) 